\title{
ON THE DESIGN OF A RELIABILITY CIRCUIT SIMULATOR
}

\author{
JAN VERWEIJ and MEINDERT LUNENBORG \\ University of Twente, MESA Research Institute, P.O. Box 217, 7500 AE Enschede, The Netherlands
}

(Receited 6 November 1992)

\begin{abstract}
This paper describes the outcome of a study into the feasibility of a reliability circuit simulator for ICs in general and the critical parameters involved in particular. The necessary conditions are formulated that have to be fulfilled before any construction of the reliability simulator is meaningful or can be done at all. It has been found that failure mechanisms in the wear-out regime meet these conditions. Next, a general approach is given to make a simulator. This approach is actually derived from circuit simulator activities on hot carrier degradation and electromigration, respectively. Finally, the different groups of parameters are defined.
\end{abstract}

\section{INTRODUCTION}

In the early days of the integrated circuit technology, each circuit was built together out of its components, i.e. transistors and resistors. This so called breadboarding was soon replaced by a computer simulation as the complexity of the circuits grew and proper models of its components became available.

So far, only the electrical performance of the circuit at the start of its life was calculated in computer simulation ( 0 -h performance). Recently, several research institutes began working on the development of a reliability circuit simulator, i.e., a simulator that takes into account the reliability aspects of semiconductor devices [1-5].

Incorporating a reliability simulator in an existing electrical circuit simulator gives designers and $\mathrm{QA}$ a tool to check the reliability performance of their design together with the zero-hour electrical performance. The aim of reliability simulation is to reduce the number of (re)design cycles and reliability tests, as sketched in Fig. 1.

With the help of a reliability circuit simulator, it is possible to avoid reliability problems due to design marginalities; this can prevent redesigns, a high workload and delayed qualification in the late phase of product development.

Another argument to use a reliability circuit simulator is that testing for reliability becomes more and more tedious with growing circuit complexity. With the decrease of failure rate limits, demonstrating reliability is costing an astronomic amount of testing time [3]. A way to work around this problem is to predict circuit reliability at the design stage.

In reliability simulation we can study distinct different levels. At the process level (i), mechanisms of materials and elementary structures are simulated $[6,7]$. At the circuit simulator level (ii), the reliability behaviour of complete circuits is simulated. At the system level (iii), systems with many ICs and com- ponents are simulated. In this article, the focus will be on reliability simulation at the circuit level (ii).

\section{NECESSARY CONDITIONS}

For each reliability group, or even each test within one group, particular failure mechanisms can be active with different impact on reliability of the circuit. Whether for each mechanism a separate reliability circuit simulator is required or can be made depends on the following conditions:

(a) Is the mechanism a bottle neck in the application?

(b) Can the mechanism be modelled properly and derated to practical use conditions?

(c) Is it possible to take proper measures during design (or processing) when the simulator shows insufficiencies?

A general approach to achieving a reliability circuit simulator is as follows: first carry out a failure analysis, followed by a categorisation of the observed mechanisms. Look for the dominant failure modes and determine the related acceleration factors. Then, construct appropriate models allowing for derating to practical use conditions. Perform parameter extraction to determine the model parameters. Finally, incorporate the results into the (electrical circuit) simulator.

In the next section different groups of reliability tests will be discussed.

\section{GROUPS OF RELIABILITY TESTS}

There are several groups of reliability tests:

(a) electrical endurance (electromigration, hot carrier degradation etc.),

(b) humidity,

(c) temperature cycling,

(d) ESD etc. 


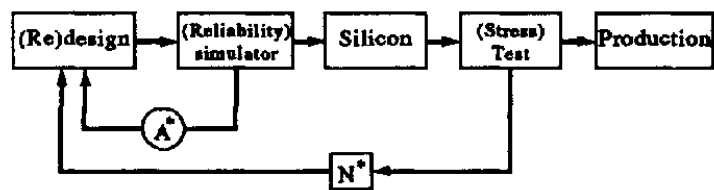

Fig. 1. The design process: the purpose of $\mathrm{A}$ is the reduction of $N$. saving costs and losses by failures.

We leave out in further considerations the mechanisms that are related to plastic packaging, i.e., the mechanisms that are active in the temperature cycling and humidity groups of tests because we feel that the conditions mentioned above are not fulfilled (derating is not possible, corrective actions are not possible in design but are in process). Therefore, we concentrate on the electrical endurance.

In the electrical endurance tests we have two important failure rate regimes, namely, early failures (carly-life) and wear-out. A well known diagram in reliability analysis is the so called bathtub diagram, as shown in Fig. 2.

In early-life, the failure rates of products under test are decreasing. During working life the number remains more or less constant, while in the end wear-out takes place and the number of failures increases again.

In Table 1, the different steps for constructing a simulator are related to the two different regimes in endurance test.

After the above steps, there is sufficient knowledge of wear-out failures to allow categorisation and modelling of key mechanisms. Now, it is the actual simulation that is missing. In practice, too little data for the early-life failure exist to even have realistic models. Therefore, only the dominant wear-out mechanisms will be discussed here (examples given are related to electrical endurance mechanisms, in this case electromigration and hot carrier effects).

\section{CRITICAL PARAMETERS FOR CIRCUIT RELIABILITY SIMULATION}

Important groups of parameters for circuit reliability simulation are summarised and discussed briefly in the following paragraphs. We have seven distinct parameter groups:

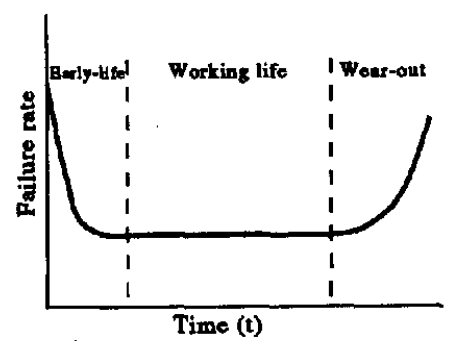

Fig. 2. Bathtub curve showing different failure rate regimes during product lifetime
Table 1. The different steps for construction of a simulator, related to early life and wearout regimes

\begin{tabular}{lcc}
\hline Step & Early life & Wear-out \\
\hline Failure analysis & + & + \\
Categorise & - & + \\
Models & - & + \\
Simulation & - & - \\
\hline
\end{tabular}

(1) electrical parameters

(2) spatial parameters

(3) stressors

(4) physical parameters

(5) progress parameters

(6) time evolution parameters

(7) spatial-dependence parameters.

1. Electrical parameters change under the influence of a stressor and also determine circuit performance. An example is the threshold voltage of an MOS transistor that changes when subjected to hot carrier effects induced by high drain voltages. Another example is the resistance $R$ of a metal stripe that changes under the influence of electromigration at high current densities.

2. Spatial parameters have an influence on circuit reliability and they are under the control of the designer. Examples are the (effective) length of an MOS transistor and the width of a metal stripe (electromigration).

3. Stressors are externally modifiable quantities that accelerate degradation. Examples are drain voltage (hot carriers) and current density (electromigration).

4. Physical parameters describe the physical nature of used materials. Examples for metallisation are grain-size and diffusion-coefficients.

5. Progress parameters describe the influence of process variations. Examples are the LDD configuration [8] (Lightly Doped Drain, for hot carriers) and copper doping of aluminium (electromigration). Together with group (5) parameters, they are used in process level or physical simulation and some can be optimised by process engineers for maximum reliability. The result of optimisation is reflected in design-rules: for metallisation in the maximum current density.

6. Time-evolution parameters describe how the electrical parameters change in time and how they depend upon group (3) parameters. They have to be derived from proper reliability experiments and they

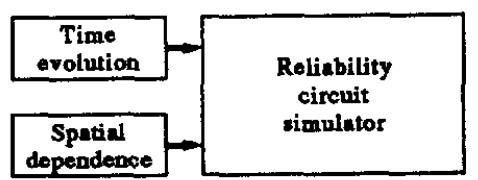

Fig. 3. The two inputs for reliability simulation. 
serve as one of the inputs for a reliability circuit simulator.

7. Spatial-dependence parameters describe how the reliability lifetime of circuit components depends on geometry (i.e., the group (2) parameters). They form the other input for a reliability circuit simulator (see Fig. 3).

\section{CONCLUSIONS}

To construct a reliability circuit simulator, it is necessary to build a proper model of the wear-out phenomena. Early life failures are difficult to model because of the little data of this regime. Critical parameters (such as time-evolution and spatialdependence parameters) then have to be determined by experiment. Finally, experimental verification of the accuracy of the simulator has to be carried out.

\section{REFERENCES}

1. S. Aur, D. E. Hocevar and P. Yang, Circuit hot electron effect simulation, IEDM Tech. Dig., 498-501 (1987).

2. P. M. Lee, M. M. Kuo, K. Seki, P. K. Ko and C. Hu, Circuit aging simulator (CAS), IEDM Tech. Dig., 134-137 (1988).

3. C. Hu, IC Reliability simulation, IEEE $J$. Solid-St. Circuits, 27, 241-246 (1992).

4. M. M. Kuo, K. Seki, P. M. Lee, J. Y. Choi, P. K. Ko and $\mathrm{C}$. Hu, Simulation of MOSFET lifetime under a.c. hot-electron stress, IEEE Trans. Electron Dev., 35 (1988).

5. W.-J. Hsu, B. J. Sheu, S. M. Cowda and C.-G. Hwang, Advanced integrated-circuit reliability simulation including dynamic stress effects, IEEE J. Solid-St. Circuits, 27, 247-257 (1992).

6. B. K. Liew, P. Fang, N. W. Cheung and C. Hu, Reliability simulator for interconnect and intermetallic connect. Proc. IRPS, pp. 111-118 (1990).

7. J. Niehof, D. C. L. van Geest and J. F. Verweij, Electromigration reliability simulator, Mat. Res. Soc. Symp. Proc. (to appear in 1992)

8. J. J. Sanchez, K. K. Hsueh and T. A. DeMassa, Drain-engineered hot-electron-resistant device structures-a review, IEEE Trans. Electron Der., 36 (1989). 